\title{
Die Verdoppelung des Verbs laa 'lassen' im Zürichdeutschen
}

\author{
Katja Schlatter Gappisch (Zürich)
}

\begin{abstract}
In some Swiss German dialects the verb laa 'let' can be doubled under certain conditions. The paper discusses the factors accounting for this variation. It investigates the syntactic environment of laa-constructions in the dialect of Zurich. The analysis of the data suggests that, first, the laa-doubling is favoured in certain constructions such as, for instance, imperative constructions. Second, the elder informants use the laa-doubling more often than the younger ones.
\end{abstract}

\section{$1 \quad$ Einleitung}

\subsection{Fragestellung}

Im Rahmen der Untersuchung des Projekts "Dialektsyntax des Schweizerdeutschen" wurde festgestellt, dass die Verdoppelung von laa nur unter bestimmten Bedingungen zu Stande kommt. ${ }^{1}$ Die Grammatiken, Sekundärliteratur und empirische Daten liefern unterschiedliche und z. T. widersprüchliche Angaben zum syntaktischen Kontext der laa-Verdoppelung. Die erforderlichen syntaktischen Rahmenbedingungen für die laa-Verdoppelung sind also noch nicht vollständig geklärt. Was steuert diese Variation? In der vorliegenden Arbeit wird mit einer empirischen Untersuchung den Fragen nachgegangen, welche syntaktischen Kontexte eine Verdoppelung begünstigen bzw. einschränken, und ob sich Hinweise auf die Funktion der Verdoppelung finden lassen. ${ }^{2}$

In nächsten Abschnitt 1.2 wird das Phänomen der Verbverdoppelung erläutert. Nach einer kurzen deskriptiven Analyse der regionalen Verteilung im Abschnitt 1.3 wird auf die aufgetretene Variation Bezug genommen (Abschnitt 1.4). Im methodischen Teil folgt die Explizierung der Fragestellungen und Hypothesen (Abschnitt 2.1). Ferner wird die Zusammenstellung des Fragebogens und die Durchführung der Befragung erläutert (Abschnitte 2.2, 2.3 und Appendix). Nach der Darstellung der erhobenen Daten wird die Bedeutung der Ergebnisse schliesslich in einem letzten Teil diskutiert. Die vorliegende Untersuchung beschränkt sich auf den zürichdeutschen Sprachraum.

\subsection{Das Phänomen der Verbverdoppelung und seine Besonderheiten}

Die Verbverdoppelung ist eine syntaktische Eigenart der Deutschschweizer Dialekte. Sie besteht darin, dass unter gewissen Bedingungen Verben, die einen Infinitiv regieren, in verkürzter Form wiederholt werden. Das wiederholte, phonetisch reduzierte Verb kommt dabei direkt vor den regierten Infinitiv zu stehen (cf. Lötscher 1993: 180; Schmidt 2000: 1). Bei den

1 Zum Projekt "Dialektsyntax des Schweizerdeutschen. Syntaktischer Atlas der Deutschen Schweiz" (SADS) siehe www.ds.uzh.ch/dialektsyntax.

2 Zur Verwendung des Terminus Verdoppelung und zum Hintergrund der Untersuchung siehe das Editorial zum vorliegenden Heft. 
Verben gaa und choo sind zwischen wiederholter Kurzform und Infinitiv eingeschobene Objekte möglich (cf. Schmidt 2000: 42). Die Verbverdoppelung tritt nur bei den Verben gaa, choo, laa und afaa auf:

(1) a. Ich gaa go tschute.

b. Ich chume cho luege.

c. Ich laa di la mache.

d. Ich faa afa ässe.

Dabei nimmt das Verb gaa eine dominante Rolle ein, in der ganzen Schweiz ist seine Verdoppelung bei Infinitivanschluss obligatorisch. ${ }^{3}$ Demgegenüber tritt die Verdoppelung von laa in weiten Teilen der Schweiz nur als Variante neben Konstruktionen ohne Verdoppelung auf (cf. Lötscher 1993: 182).

\subsection{Regionale Verteilung}

Die Daten des Zürcher SADS-Projekts zeigen bezüglich der Verdoppelung von laa eine klare regionale Verteilung: Im westlichen Teil der Schweiz wird verdoppelt, im Osten wird nicht verdoppelt. Dabei stellt der Kanton Zürich eine Grenzregion dar. Während im südlichen Teil des Kantons die Verdoppelung auftritt, ist dies nördlich der Stadt Zürich kaum der Fall (s. Karte 1 im Appendix). ${ }^{4}$ Dass der Kanton Zürich eine Übergangszone bildet, zeigt sich auch darin, dass in keiner einzigen Zürcher Ortschaft zu 100\% verdoppelt wird. Die höchsten Werte finden sich in Bauma (62,5\%), Egg und Grüt (je $40 \%$ ), Stallikon und Hedingen (je $37,5 \%$ ) und Rifferswil, Thalwil und Meilen (je 33,3\%).5 Von den insgesamt 42 getesteten Ortschaften tritt nur in 26 überhaupt eine Verdoppelung auf. Von 315 im Kanton Zürich befragten Personen haben lediglich 51 eine Verdoppelungskonstruktion verwendet.

Ein ähnliches Bild zeigt der Schweizerdeutsche Sprachatlas (SDS). Auch hier wird Verdoppelung für südliche Zürcher Ortschaften notiert (SDS III 263): in Langnau, Horgen, Wädenswil, Meilen, Hinwil und Bäretswil (s. Karte 2 im Appendix nach Lötscher 1993). Auffällig sind die ausgebliebenen Verdoppelungen im Knonauer Amt. Der SDS-Atlas dokumentiert also ein noch eingeschränkteres Verdoppelungsgebiet.

Diese Analysen lassen darauf schliessen, dass die Mehrheit im Kanton Zürich nicht verdoppelt und dass im südlichen Teil des Kantons häufiger verdoppelt wird als im Norden.

\subsection{Variation}

Die Abfrage des SADS-Projekts zeigt eine grosse Variation in der Verwendung von Verdoppelungskonstruktionen. In den Gegenden, in denen die laa-Verdoppelung grundsätzlich möglich ist, wird sie nicht konsequent verwendet. Was steuert diese Variation? Was ist ihre Funktion?

Zum Einen scheint es der syntaktische Kontext zu sein, der die Varianten selegiert. In der SADS-Übersetzungsaufgabe II.3 mit dem Verb laa in einer Präsensform (Er lässt den Schreiner kommen) wird bedeutend mehr verdoppelt als in den Aufgaben II.1 (Hast du die Uhr flicken lassen?) und II.5 (Ihr dürft alles liegen lassen), in denen laa im Perfekt oder als Infinitiv abhängig von einem Modalverb steht. Dieser Befund stimmt im Grossen und Ganzen mit den syntaktischen Rahmenbedingungen überein, die nach Lötscher (1993: 181) für die Wiederholung von laa erforderlich sind: Die flektierte Form des Verdoppelungsverbs sollte in

3 Die Verdoppelung von gaa kann als einzige Verdoppelung sogar ausserhalb des schweizerdeutschen Sprachraums im Oberelsässischen und Südbadischen belegt werden (cf. Lötscher 1993: 182).

4 Verdoppelung trat hier lediglich bei einer Person aus Niederwenigen und einer aus Bassersdorf auf.

5 Diese Angaben beziehen sich auf die Übersetzung des Satzes II.3 Er lässt den Schreiner kommen aus dem SADS-Fragebogen. 
Zweitstellung stehen, d. h. das Verb laa darf nicht im Perfekt oder in einer Modalkonstruktion am Ende des Satzes stehen. Allerdings scheint es sich dabei nicht um eine allgemein gültige normative Regel zu handeln. Die in der zürichdeutschen Grammatik aufgeführten Beispiele für die Verdoppelung von laa erfüllen diese Bedingungen nämlich gerade nicht (cf. Weber 1948: 247):

(2) a. Las es nüd la fale!

b. Wottsch mi ächt la gaa laa!

c. Er hät en en Tokter la studiere laa.

In keinem der Beispiele Webers steht die flektierte Form von laa in Zweitstellung. Auch in der SADS-Datenbank ist ein Widerspruch zu Lötschers Rahmenbedingungen zu finden: In der Übersetzungsaufgabe II.5 Ihr dürft alles liegen lassen verwendeten einige Testpersonen eine Imperativkonstruktion Lönd alles la ligge bzw. Lönd alles ligge. Dabei wählte die Mehrheit von ihnen die Option mit Verdoppelung. Die Verbverdoppelung scheint also auch möglich zu sein, wenn laa nicht in Zweitstellung sondern am Satzanfang steht. Es ist also noch nicht hinreichend geklärt, in welchen syntaktischen Kontexten die laa-Verdoppelung möglich ist. Aus den Befunden der Datenbankanalyse könnte eine neue syntaktische Rahmenbedingungen für die Verdoppelung des Verbs laa abgeleitet werden: Das Verdoppelungsverb muss in finiter Form stehen.

Eine weitere syntaktische Begründung findet sich in der Arbeit von Schmidt (2000). Sie hat in ihrer Untersuchung festgestellt, dass in Konstruktionen, in denen die flektierte Form von laa direkt auf den abhängigen Infinitiv treffen würde, vermehrt verdoppelt wird. Zu einem solchen Aufeinandertreffen kommt es unter anderem in Nebensatzkonstruktionen. Vgl. (3a) und (3b) aus dem Zürichdeutschen in Schmidt (2000: 43):

(3) a. [si] lönd sich am taatort überrasche

b. $\quad$ wän er schwaarz laat $l a$ schaffe

Hier könnte laut Schmidt (2000) die Verbverdoppelung als Strategie dienen, um das scheinbar unerwünschte Zusammenkommen von finiter laa-Form und abhängigem Infinitiv zu vermeiden. Dies scheint ein interessanter Ansatz zu sein, der weiter überprüft werden sollte.

Zum Anderen könnte dieWahl der Verdoppelungskonstruktion mit dem Alter der Sprecher zusammenhängen. Beispielsweise stellt Schmidt (2000) fest, dass jüngere Gewährspersonen weniger Verdoppelungen verwendeten als ältere. Möglicherweise zählt die Verdoppelung von laa zu den syntaktischen Phänomenen, die infolge Sprachwandels immer seltener auftreten.

\section{Methoden und Vorgehensweise}

\subsection{Ziel der Befragung und Hypothesen}

Die Verdoppelung von laa ist im Zürichdeutschen fakultativ. Frühere Untersuchungen haben gezeigt, dass eine Person sogar im selben Satz Verdoppelungskonstruktionen neben Formulierungen ohne Verdoppelung verwenden kann (cf. Schmidt 2000: 39). Ziel dieser Befragung ist es, Aufschlüsse darüber zu finden, worauf die Variation gründet. Insbesondere soll ermittelt werden, in welchen syntaktischen Kontexten die Verbverdoppelung bevorzugt wird. Aus dem bisher Besprochenen lassen sich folgende Fragen und Hypothesen formulieren:

Frage 1: Welche syntaktischen Kontexte begünstigen die Verdoppelung von laa und welche schränken sie ein?

Frage 2: Welche Funktion hat die laa-Verdoppelung?

Hypothese 1: Das Verb laa wird nur verdoppelt, wenn es die Stellung des finiten Hauptverbs einnimmt. 
Hypothese 2: Steht laa in einer untergeordneten Nebensatzkonstruktion, tritt die Verdoppelung häufiger auf als in Hauptsatzkonstruktionen.

Hypothese 3: Ältere Personen verwenden häufiger die laa-Verdoppelung als jüngere.

\subsection{Fragebogen}

Für die vorliegende Arbeit wurden die Daten in Anlehnung an das SADS-Projekt mittels Fragebogen erhoben. Die Informanten wurden gebeten, Sätze aus dem Hochdeutschen in ihren Dialekt zu übersetzen.

Bei der Auswertung der Ergebnisse muss allerdings berücksichtigt werden, dass die Antwortsätze auf Dialekt durch die standarddeutschen Mustersätze beeinflusst sein können. Es ist also anzunehmen, dass die Testpersonen in der Befragung eher weniger verdoppeln, als sie es unter normalen (nicht Test-)Umständen tun würden. Umgekehrt bekommt eine verwendete Verdoppelungskonstruktion besonderes Gewicht, da sie sich trotz der standarddeutschen Vorlage durchgesetzt hat (cf. Glaser 2000: 263).

Die Gestaltung des Fragebogens lehnt sich stark an die Vorlage des Zürcher SADS-Projekts an. In dieser schweizweiten Umfrage wurden auch Daten zur laa- Verdoppelung erhoben. Die entsprechenden drei Übersetzungsaufgaben wurden in der erneuten Umfrage direkt übernommen. ${ }^{6}$ Ergänzend dazu enthält der Fragebogen Übersetzungsaufgaben mit dem Verb laa im Nebensatz und im Imperativ. Um sicher zu gehen, dass die Verdoppelung (aus semantischen oder phonologischen Gründen) nicht vom jeweiligen lexikalischen Verb abhängt, wurde zusätzlich ein Verb in allen verschiedenen Satzformen getestet (s. Aufgaben 6-10 im Fragebogen im Appendix). Der ganze Fragebogen befindet sich im Anhang. Folgende Fälle konnten also mit je zwei Übersetzungsfragen untersucht werden:

1. Das Verb laa steht im Präsens.

2. Das Verb laa steht im Imperativ.

3. Das Verb laa steht im Perfekt.

4. Das Verb laa steht im Infinitiv abhängig von einem Modalverb.

5. Das Verb laa steht im Präsens in einer Nebensatzkonstruktion.

In den Fällen 1, 2 und 5 nimmt das Verb laa die Stelle des finiten Hauptverbs ein, hier ist eine Verdoppelung zu erwarten, in den Fällen 3 und 4 eher nicht. Am häufigsten wird die Verbverdoppelung im Fall 5 erwartet (vgl. Hypothesen 1 und 2).

\subsection{Durchführung der Befragung}

Da der zürichdeutsche Sprachraum ein Grenzgebiet bezüglich der Verdoppelung von laa zu sein scheint (s. Abschnitte 1.3 und 1.4), war die regionale Herkunft der Gewährspersonen für die Auswahl der Stichprobe zentral. Die Daten sollten nur bei Personen erhoben werden, die aus einer Gegend im Kanton Zürich stammen, in der verdoppelt wird. Bei der Auswahl möglicher Gebiete stützte ich mich auf das Verteilungsbild des SADS-Projekts (s. Karte 1 im Anhang). Die Ortschaften mit der höchsten Verdoppelung liegen im Knonauer Amt, um den Zürichsee und im Zürcher Oberland. In den Zürcher Mundarten des Oberlandes, des Amtes und in den See-Mundarten scheint die Verbverdoppelung also am ausgeprägtesten zu sein. Für die Dialekte von See und Amt ist dies nicht überraschend, liegen sie doch in der westlichen Kantonshälfte. Laut Weber (1948: 21) ist insbesondere im südwestlichen Amt der Einschlag der angrenzenden Zuger Mundart (diese weist eine starke Verbverdoppelung auf)

6 Es handelt sich dabei um die Fragen 1, 3 und 5 aus dem zweiten Fragebogen. In der vorliegenden Untersuchung sind sie als die Fragen 1,2 und 4 in den Fragebogen integriert (siehe Appendix). 
festzustellen. Dass auch im eher östlich gelegenen Zürcher Oberland verdoppelt wird, mag daran liegen, dass in dieser Gegend ein relativ konservativer Dialekt bewahrt wurde (cf. Weber 1948: 20).

Für die Befragung wurden sechs Ortschaften aus dem Knonauer Amt und der Seeregion ausgewählt. Nach Möglichkeit habe ich versucht, Alter und Geschlecht der auszuwählenden Personen zu variieren. Die grösste Schwierigkeit war, dass die meisten der Angefragten Eltern aus anderen Dialektgebieten hatten. Ich habe darauf geachtet, dass mindestens ein Elternteil den betreffenden Dialekt sprach, in einzelnen Fällen sprechen jedoch beide Eltern eine andere Zürcher Mundart.

Die ersten Informanten wurden durch Hinweise aus dem Bekanntenkreis gefunden. Sie wurden jeweils telefonisch angefragt, ob sie bereit wären, an der Erhebung teilzunehmen. Danach haben sie den Fragebogen, einschliesslich frankiertem Rücksende-Couvert, per Post zugeschickt bekommen. Am Telefon wurden die Informanten jeweils nach weiteren ortsansässigen Personen gefragt. Dadurch war es meist möglich, pro Ortschaft mindestens drei Testpersonen ausfindig zu machen. Die Informanten zeigten sich grössten Teils sehr interessiert und erklärten bereitwillig ihre Teilnahme. Von 20 verschickten Fragebogen kamen 18 zurück. Die Stichprobe setzt sich folgendermassen zusammen:

\begin{tabular}{|l|l|r|}
\hline Region & Ortschaft & Anzahl GP \\
\hline Knonauer Amt & Rifferswil & 3 \\
& Mettmenstetten & 3 \\
& Stallikon & 2 \\
& Hausen a. A. & 1 \\
\hline \multirow{2}{*}{ Seeregion } & Oberrieden & 3 \\
& Wädenswil & 4 \\
& Hombrechtikon & 2 \\
\hline Total & & 18 \\
\hline
\end{tabular}

Tab. 1: Zusammensetzung der Stichprobe

Aus dem Knonauer Amt wurden die Dialekte von Rifferswil und Stallikon untersucht, die im SADS-Projekt eine gute Verdoppelungsquote zeigten. Dazu kam die Mundart aus dem westlich gelegenen Mettmenstetten. Zusätzlich wurde eine Person aus Hausen a. A. befragt. Die linksufrige Seeregion ist durch die Dialekte von Wädenswil und Oberrieden vertreten. Für Oberrieden liegen keine früher erhobenen Werte vor, dafür für das benachbarte Thalwil. Aus der rechten (östlichen) Seeregion wurde die Mundart von Hombrechtikon gewählt. Insgesamt haben neun Männer und neun Frauen an der Befragung teilgenommen. 


\begin{tabular}{|c|c|c|c|c|c|c|c|c|c|c|c|c|c|}
\hline & Ortschaft & Ge. & Jahrg. & 1.Präs & 1.Imp & 1.NS & 1.Perf & 1. Mod & 2.Präs & 2.Imp & 2.NS & 2.Perf & 2.Mod \\
\hline 1 & Rifferswil & $\mathrm{m}$ & 1936 & ja & ja & ja & z.fug & z.fug. & ja & nein & ja & z.fug. & z.fug. \\
\hline 2 & Rifferswil & $\mathrm{m}$ & 1936 & ja & nein & and. & z.fug. & z.fug. & nein & nein & ja & z.fug. & z.fug. \\
\hline 3 & Rifferswil & $\mathrm{m}$ & 1943 & nein & ja & and. & z.fug. & z.fug. & ja & nein & ja & z.fug. & z.fug. \\
\hline 4 & Mettmenst. & $\mathrm{w}$ & 1986 & nein & nein & z.pet. & z.pet. & z.pet. & nein & nein & z.pet. & z.pet. & z.pet. \\
\hline 5 & Mettmenst. & $\mathrm{m}$ & 1988 & nein & nein & & z.pet. & z.pet. & nein & nein & z.pet. & z.pet. & z.pet. \\
\hline 6 & Mettmenst. & $\mathrm{m}$ & 1962 & ja & nein & z.pet. & z.pet.* & z.pet. & nein & nein & z.pet. & z.pet. & z.pet. \\
\hline 7 & Stallikon & w & 1954 & nein & and. & and. & and. & z.pet. & nein & nein & z.pet. & z.pet. & z.pet. \\
\hline 8 & Stallikon & $\mathrm{m}$ & 1931 & ja & ja & ja & z.fug. & z.fug. & ja & ja & & z.fug. & z.fug. \\
\hline 9 & Hombrecht. & $\mathrm{m}$ & 1938 & $\mathrm{ja}$ & ja & z.pet. & z.fug. & z.pet. & nein & nein & z.pet. & z.pet. & z.pet. \\
\hline 10 & Hombrecht. & w & 1968 & ja & nein & z.pet. & z.pet. & and. & ja & nein & z.pet. & z.pet. & z.pet. \\
\hline 11 & Oberrieden & w & 1934 & ja & ja & and. & z.pet. & and. & nein & nein & z.pet. & z.pet. & and. \\
\hline 12 & Oberrieden & w & 1942 & nein & nein & and. & z.pet. & and. & nein & nein & z.pet. & z.pet. & z.pet. \\
\hline 13 & Oberrieden & w & 1970 & nein & nein & z.pet. & z.pet. & z.pet. & nein & nein & z.pet. & z.pet. & z.pet. \\
\hline 14 & Wädenswil & w & 1932 & nein & nein & Z.pet. & Z.fug. & z.pet. & nein & nein & ja & z.pet. & z.pet. \\
\hline 15 & Wädenswil & w & 1971 & ja & ja & ja & z.fug. & z.fug. & ja & ja & ja & z.fug. & z.pet. \\
\hline 16 & Wädenswil & $\mathrm{m}$ & 1934 & ja & nein & ja & z.fug. & & nein & nein & ja & z.fug. & z.fug. \\
\hline 17 & Wädenswil & $\mathrm{m}$ & 1942 & nein & nein & ja & z.pet. & z.pet. & nein & nein & z.pet. & z.pet.* & z.pet. \\
\hline 18 & Hausen a. A. & $\mathrm{w}$ & 1971 & ja & and. & and. & z.pet. & z.pet. & nein & nein & ja & z.pet. & z.pet. \\
\hline
\end{tabular}

Tab. 2: Gesamtübersicht der Antworten; Ge. = Geschlecht, NS = Nebensatz, Imp. = Imperativ, Perf. = Perfekt, Mod $=$ Modalkonstruktion, and. $=$ andere Konstruktion, * Partizip glaa verwendet

\section{$3 \quad$ Resultate und Vergleich}

In diesem Abschnitt werden die Ergebnisse in Bezug auf Region (Abschnitt 3.1), syntaktischen Kontext (Abschnitt 3.2), Wortfolge (Abschnitt 3.3) und Alter der Informanten (Abschnitt 3.4) dargestellt und ausgewertet. Die Interpretation der Resultate folgt im Abschnitt 4.

\section{1 Übersicht und Auswertung nach Regionen}

Zuerst werden die Antworten zu den aus dem Zürcher Dialektsyntax-Projekt übernommenen Aufgaben und den zwei Ergänzungsfragen (d. h. die Aufgaben 1-5 aus dem Fragebogen) aufgelistet. Sie sind als erste (1.) Präsens-, Imperativ-, Nebensatz-, Perfekt- und Modalkonstruktion bezeichnet. Mit dem Zusatz (2.) folgen die Antworten zu den Aufgaben mit dem Verb liegen (Aufgaben 6-10). Verbverdoppelungen werden jeweils mit "ja" gekennzeichnet, für unbrauchbare Antworten (meist keine Formulierung mit laa) wird ein "and." (anders) gesetzt. Bei den Nebensatz-, Perfektund Modalkonstruktionen ohne Verdoppelung wird angegeben, ob die zentrifugale (z.fug.), z. B. la ligge, oder die zentripetale (z.pet.), z. B. ligge la, Wortfolge verwendet worden ist.

Die Verdoppelungskonstruktionen zeigen auf den ersten Blick keine grossen Regelmässigkeiten in ihrem Auftreten. Ihr Auftreten erstreckt sich über alle Beispiele, in denen die Verdoppelung erwartet werden konnte, in einem Fall kommt die Verdoppelung sogar in einer Modalkonstruktion vor (s. Nr. 16 Wädenswil 1.Mod in der Tab. 2). Nur drei Personen haben überhaupt nie verdoppelt (Nr. 4, 12 und 13 in der Tab. 2). In der Aufgabe zur ersten Nebensatzkonstruktion (1.NS) fällt die grosse Anzahl unbrauchbarer Antworten auf.

Eine Auswertung nach Region zeigt, dass in jeder Ortschaft Verdoppelungen aufgetreten sind: 


\begin{tabular}{|l|c|c|c|c|c|c|c|c|}
\hline \multirow{2}{*}{ Ortschaft } & \multicolumn{2}{|c|}{ Präsens } & \multicolumn{2}{c|}{ Imperativ } & \multicolumn{2}{c|}{ Nebensatz } & \multicolumn{2}{c|}{ Total } \\
\cline { 2 - 9 } & VV/bb. & $\approx \%$ & VV/bb. & $\approx \%$ & VV/bb. & $\approx \%$ & VV/bb. & $\approx \%$ \\
\hline Riffersw. 3 & $4 / 6$ & 66.7 & $2 / 6$ & 33.3 & $4 / 4$ & 100 & $10 / 16$ & 62.5 \\
Mettmen. 3 & $1 / 6$ & 16.7 & $0 / 6$ & 0 & $1 / 6$ & 16.7 & $2 / 18$ & 11.1 \\
Stallikon 2 & $2 / 4$ & 50 & $2 / 3$ & 66.7 & $2 / 3$ & 66.7 & $6 / 10$ & 60 \\
Hombrecht. 2 & $3 / 4$ & 75 & $1 / 4$ & 25 & $0 / 4$ & 0 & $4 / 12$ & 33.3 \\
Oberried. 3 & $1 / 6$ & 16.7 & $1 / 6$ & 16.7 & $0 / 4$ & 0 & $2 / 16$ & 12.5 \\
Wädensw.4 & $3 / 8$ & 37.5 & $2 / 8$ & 25 & $6 / 8$ & 75 & $11 / 24$ & 45.8 \\
Hausen 1 & $1 / 2$ & 50 & $0 / 1$ & 0 & $1 / 1$ & 100 & $2 / 4$ & 50 \\
\hline Total 18 & $15 / 36$ & 41.7 & $8 / 34$ & 23.5 & $14 / 30$ & 46.7 & $37 / 100$ & 37 \\
\hline
\end{tabular}

Tab. 3: Auswertung der laa Verdoppelung nach Ortschaften; VV = Verbverdoppelung, bb. = brauchbar

Die Zahl nach dem Ortsnamen gibt jeweils an, wie viele Personen pro Ortschaft an der Befragung teilgenommen haben. Da diese Anzahl variiert, sind die Ergebnisse nur bedingt vergleichbar. So gehen beispielsweise die hohen $50 \%$ in Hausen a. A. auf eine einzelne Teilnehmerin zurück (s. Tab. 3). Auffallend sind die hohen Werte in Rifferswil und Stallikon. Während in letzterem jedoch eine Person gar nicht verdoppelte, die andere dafür sehr stark, zeigen alle drei Informanten von Rifferswil eine deutliche Tendenz zur Verdoppelung. Beachtenswert sind auch die Ergebnisse aus Wädenswil: alle vier Gewährspersonen verwendeten hier mindestens eine Verdoppelungskonstruktion, eine Informantin hat in allen erwarteten Fällen (also im Präsens, Imperativ und Nebensatz) verdoppelt, ein Informant hat zudem in einer Formulierung mit Modalverb verdoppelt (s. auch Nr. 14-17 in Tab. 2). Eine tiefe Verdoppelungsquote wird in Oberrieden registriert. Von drei Testpersonen haben zwei nicht verdoppelt (s. auch Nr. 11-13 in Tab. 2). Der geringste Anteil an Verdoppelungen findet sich in Mettmenstetten. Von drei Personen zeigen eine gar keine und zwei nur je eine Verbverdoppelung. In Wädenswil sind es eigentlich total $12 \mathrm{VV}$ statt 11 in der Tabelle 3, da dort zusätzlich in einer Modalkonstruktion eine VV aufgetreten ist.

Es fällt auf, dass die Verdoppelungen ungleich auf die verschiedenen Satzarten verteilt sind. Dieser Befund wird im nächsten Abschnitt ausführlicher dargestellt.

\subsection{Auswertung des syntaktischen Kontexts}

Tabelle 4 zeigt, in welchen syntaktischen Kontexten Verdoppelungen aufgetreten sind. Die laa-Verbverdoppelung tritt hauptsächlich in Präsens-, Imperativund Nebensatzkonstruktionen auf, also in Formulierungen mit laa in der Stellung des finiten Hauptverbs. Eine Überraschung gibt es dennoch: ein Informant verdoppelt in einer Formulierung mit Modalverb: (6) Ihr törfed alles la ligge la. Es handelt sich dabei um einen Mann mit Jahrgang 1934, wohnhaft und aufgewachsen in Wädenswil. Sein Vater sprach ebenfalls den Dialekt von Wädenswil, beim Dialekt der Mutter steht ein Strich, was vermuten lässt, dass diese keinen Schweizer Dialekt gesprochen hat oder dass der Informant ohne seine Mutter aufgewachsen ist.

\begin{tabular}{|l|r|r|r|r|r|r|}
\hline & \multicolumn{2}{|c|}{ Verdoppelung } & \multicolumn{2}{c|}{ keine Verdopp. } & \multicolumn{2}{c|}{ brauchbar } \\
\cline { 2 - 7 } & Anz. & \multicolumn{1}{c|}{$\approx$} & \multicolumn{1}{c|}{ Anz. } & \multicolumn{1}{c|}{$\%$} & \multicolumn{1}{c|}{ Anz. } & $\approx \%$ \\
\hline Präsens & 15 & 41.7 & 21 & 58.3 & 36 & 100 \\
Imperativ & 8 & 23.5 & 26 & 76.5 & 34 & 100 \\
Nebensatz & 14 & 46.7 & 16 & 53.3 & 30 & 100 \\
Perfekt & 0 & 0 & 35 & 100 & 35 & 100 \\
Modalverb & 1 & 3.1 & 31 & 96.9 & 32 & 100 \\
\hline
\end{tabular}

Tab. 4: Auswertung der Fragebogen nach grammatischem Kontext 
Weiter interessant ist, dass Informant 6 aus Mettmenstetten und Informant 17 aus Wädenswil je in einem Perfektsatz das morphologisch markierte Partizip glaa verwenden. ${ }^{7}$

Vergleicht man die drei Satzkonstruktionen mit laa in der Stelle des Hauptverbs miteinander (s. Tab. 4), zeigt sich die höchste Verdoppelungsquote in der Nebensatzkonstruktion $(46,7 \%)$, nur geringfügig tiefer ist die Quote im Hauptsatz $(41,7 \%)$. Zu berücksichtigen ist dabei, dass bei den Aufgaben mit einem Nebensatz einige ungültige Antworten geliefert wurden (z. B. Antwortsätze, die keinen Nebensatz beinhalteten). Die Ergebnisse der zwei Konstruktionsarten können also nur bedingt miteinander verglichen werden. Klar weniger Verdoppelungen werden in Imperativsätzen verwendet $(23,5 \%)$.

Auffallend ist die grosse Variation innerhalb der einzelnen Fragebogen, also die intrapersonale Variation. Es ist keineswegs so, dass wer einmal verdoppelt, immer verdoppelt. Nur zwei Personen haben in allen sechs Aufgaben mit laa in der Stellung des Hauptverbs verdoppelt (s. Nr. 8 Stallikon und 15 Wädenswil in Tab. 2). Interessanterweise treten in den ersten Aufgaben mit Präsens und Imperativkonstruktionen (Aufgabe 3 und 4 des Fragebogens) bedeutend mehr Verbverdoppelungen auf als in den entsprechenden Aufgaben 7 und 10 am Ende des Fragebogens. In dieser zweiten Serie beinhalten alle Übersetzungsvorgaben eine Formulierung mit dem Verb ligge.

Tabelle 5 zeigt, dass die Verbverdoppelung in Präsens- bzw. Imperativsätzen mit den Verben choo oder sii doppelt bis dreimal so oft auftritt. Sechs Gewährspersonen haben nur im Präsenssatz mit choo verdoppelt, vier Personen in beiden Sätzen und eine Person hat nur im Präsenssatz mit ligge eine Verdoppelung verwendet. Bei den Imperativsätzen haben vier Gewährspersonen lediglich mit dem Verb sii verdoppelt, zwei haben in beiden Sätzen die Verdoppelung verwendet. Bei den Nebensatzkonstruktionen ist das Verhältnis ausgeglichener, vier Personen verdoppeln in beiden Aufgaben, zwei nur im Nebensatz mit choo und vier nur im Nebensatz mit ligge. Allerdings ist anzumerken, dass diese vier letzten Personen in der Aufgabe zu choo eine ungültige Formulierung verwendet haben. ${ }^{8}$

\begin{tabular}{|l|r|c|r|r|}
\hline & \multicolumn{2}{|c|}{ Verdoppelung } & \multicolumn{2}{c|}{ brauchbar } \\
\cline { 2 - 5 } & Anz. & \multicolumn{1}{c|}{$\approx$} & \multicolumn{1}{c|}{ Anz. } & $\approx \%$ \\
\hline Präsens + choo & 10 & 55.6 & 18 & 100 \\
Präsens + ligge & 5 & 27.8 & 18 & 100 \\
Imperativ + sii & 6 & 37.5 & 16 & 100 \\
Imperativ + ligge & 2 & 11.1 & 18 & 100 \\
Nebensatz + choo & 6 & 50 & 12 & 100 \\
Nebensatz + ligge & 8 & 44.4 & 18 & 100 \\
\hline
\end{tabular}

Tab. 5: Auswertung der Fragebogen nach Satzart und Verben

\subsection{Auswertung der Wortabfolge}

In den Nebensatz-, Perfekt- und Modalkonstruktionen ohne Verbverdoppelung sind zwei verschiedene Wortabfolgen festzustelllen: die zentrifugale Wortfolge, bei der das Verb laa dem abhängigen Infinitiv vorangeht (z. B. la ligge) und die zentripetale mit nachgestelltem laa (z. B. ligge la). Die Auswertung des Fragebogens zeigt, dass die zentripetale Wortfolge klar dominiert.

Es fällt auf, dass die zwei Abfolgemuster im Perfekt ungleich auf die Beispielsätze verteilt sind. Im Satz mit dem Verb flicke werden die zwei Abfolgemuster fast ausgeglichen verwen-

\footnotetext{
${ }^{7}$ Diese zwei Fälle sind in der Übersichtstabelle (Tab. 2) mit * gekennzeichnet.

${ }^{8}$ Zur Ursache dieser grossen Anzahl ungu“ltiger Antworten vgl. Abschnitt 4.5.
} 
det (s. Tab. 6). Bei dem Verb ligge überwiegt dagegen die zentripetale Wortfolge klar (ligge la). In den Nebensätzen ohne Verbverdoppelung war ausschliesslich das zentripetale Abfolgemuster anzutreffen. Gewährspersonen, die allgemein die zentrifugale Wortfolge verwenden, haben in den Nebensätzen verdoppelt.

\begin{tabular}{|l|r|r|r|r|r|r|}
\hline \multirow{2}{*}{ Formulierung } & \multicolumn{2}{|c|}{ WF zentrifugal } & \multicolumn{2}{c|}{ WF zentripedal } & \multicolumn{2}{c|}{ brauchbar } \\
\cline { 2 - 7 } & \multicolumn{1}{c|}{ Anz. } & \multicolumn{1}{c|}{$\approx \boldsymbol{\%}$} & \multicolumn{1}{c|}{ Anz. } & \multicolumn{1}{c|}{$\approx \boldsymbol{\%}$} & \multicolumn{1}{c|}{ Anz. } & \multicolumn{1}{c|}{ \% } \\
\hline Perfekt Total & 14 & 40 & 21 & 60 & 35 & 100 \\
Perfekt + flicke & 8 & 47.1 & 9 & 52.9 & 17 & 100 \\
Perfekt + ligge & 6 & 33.3 & 12 & 66.7 & 18 & 100 \\
\hline Modalverb Total & 10 & 32.3 & 21 & 67.7 & 31 & 100 \\
dürfen + ligge & 5 & 35.7 & 9 & 64.3 & 14 & 100 \\
können + ligge & 5 & 29.4 & 12 & 70.6 & 17 & 100 \\
\hline Nebensatz Total & 0 & 0 & 16 & 100 & 16 & 100 \\
Total & 24 & 36.4 & 42 & 63.6 & 66 & 100 \\
\hline
\end{tabular}

Tab. 6: Wortfolge (WF) in Nebensatz-, Perfekt- und Modalkonstruktionen

Die Resultate in der Tabelle 6 zusammen mit den Resultaten in der Tabelle 2 lassen eine geringe intrapersonale Variation erkennen. Die Gewährspersonen verwenden ziemlich konsequent nur jeweils eineWortfolge. Lediglich drei Personen variieren in je einem Fall das Abfolgemuster. Sechs Personen (das ist ein Drittel der Stichprobe) verwenden (fast) ausschliesslich die zentrifugale Wortfolge, dies sind gleichzeitig die Gewährspersonen, die in der Untersuchung am häufigsten verdoppelt haben. Sie sprechen die Dialekte von Rifferswil, Stallikon und Wädenswil.

\subsection{Auswertung nach Altersgruppen}

Die Stichprobe umfasst Jahrgänge von 1931 bis 1988. Da lediglich zwei Gewährspersonen sehr jung sind (unter 30 Jahren), werden nur zwei Altersgruppen gebildet. Diese setzen sich aus folgenden Jahrgängen zusammen:

Gruppe 1: $\quad$ 1931, 1932, 1934, 1936, 1938, 1942, 1943

Gruppe 2: $\quad$ 1954, 1962, 1968, 1970, 1971, 1986, 1988

Wie erwartet verwendet die ältere Generation häufiger Verbverdoppelungen. Während das Verhältnis zwischen Konstruktionen mit Verbverdoppelung (VV) und solchen ohne bei den Aufgaben im Präsens fast ausgeglichen ist (Gr. 1: 31\% vs. Gr. 2: $27 \%$ ) verlagert es sich bei den Nebensatzbeispielen (Gr. 1: 38,5\% vs. Gr. 2: 22,2 \%) und bei den Imperativsätzen (Gr. 1: $23 \%$ vs. Gr. 2: 12,5\%) deutlich zugunsten der Gruppe 1 (s. Abb. 1). Diese verdoppelt hier beinahe zweimal so oft. Bemerkenswert ist allerdings, dass in Gruppe 2 mit jüngeren Jahrgängen eine Informantin mit Jahrgang 1971 in allen sechs Präsens-, Imperfekt- und Nebensatzbeispielen verdoppelt hat. 


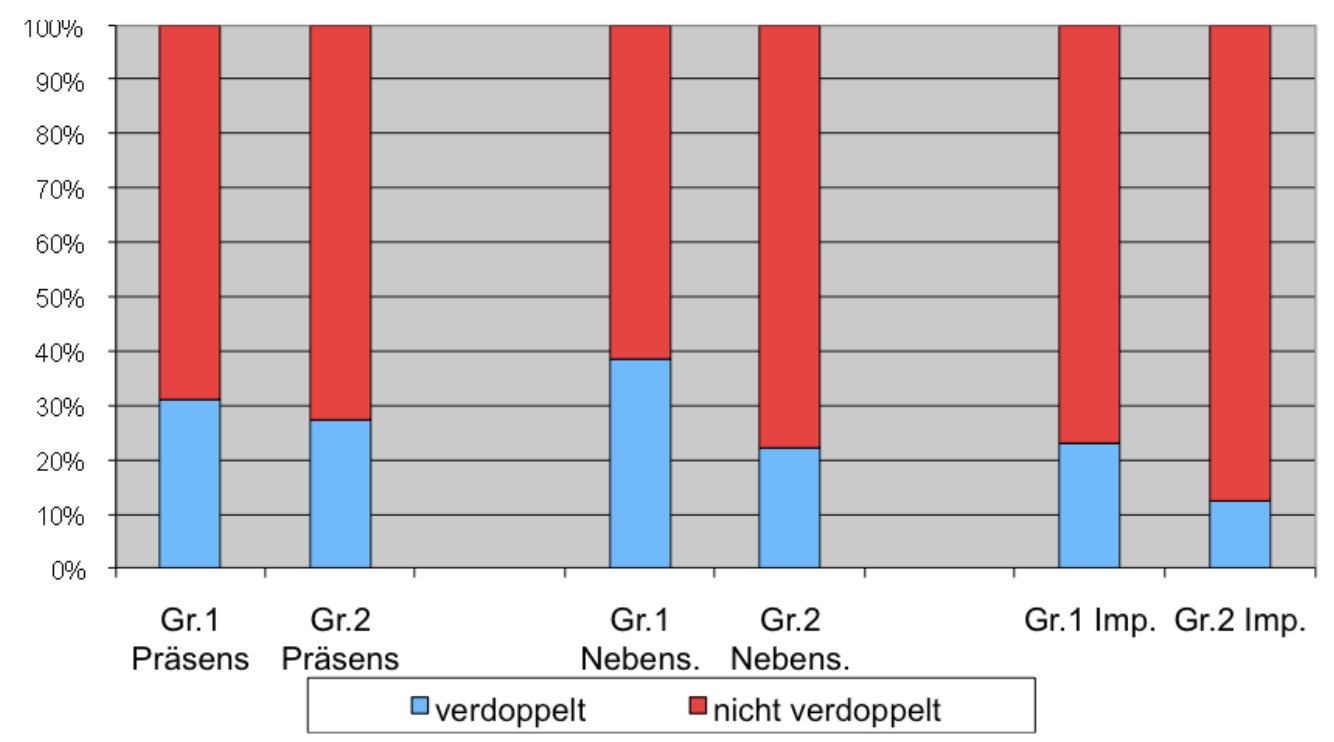

Abb. 7: Auswertung nach Altersgruppen

\section{Diskussion}

Im Folgenden werden die Ergebnisse mit Bezug auf die in den Abschnitten 1.1 und 2.1 aufgeführten Fragestellungen und Hypothesen diskutiert. Das Hauptanliegen der vorliegenden Arbeit ist, der Frage nachzugehen, wodurch die Variation der laa-Verdoppelung gesteuert wird. Zur Erinnerung sind hier die Fragen und Hypothesen aus dem Abschnitt 2.1 noch einmal aufgeführt:

Frage 1: Welche syntaktischen Kontexte begünstigen die Verdoppelung von laa und welche schränken sie ein?

Frage 2: $\quad$ Welche Funktion hat die laa-Verdoppelung?

Hypothese 1: Das Verb laa wird nur verdoppelt, wenn es die Stellung des finiten Hauptverbs einnimmt.

Hypothese 2: Steht laa in einer untergeordneten Nebensatzkonstruktion, tritt die Verdoppelung häufiger auf als in Hauptsatzkonstruktionen.

Hypothese 3: Ältere Personen verwenden häufiger die laa-Verdoppelung als jüngere.

\subsection{Variation aufgrund des syntaktischen Kontexts}

Die Resultate, wie im Abschnitt 3 dargestellt, entsprechen weitgehend der aufgestellten Hypothese 1: Die Wiederholungsstruktur wird verwendet, wenn laa die Stellung des finiten Hauptverbs einnimmt. ${ }^{9}$ Dass ein Informant in einer Modalkonstruktion verdoppelt hat, deutet jedoch darauf hin, dass es sich dabei eher um eine Tendenz als um eine obligatorische Regel handelt. Bedenkt man die eingesetzte Befragungsmethode, bekommt dieses Einzelergebnis zusätzliche Bedeutung: Der Informant hat seine Konstruktion entgegen der Struktur der standarddeutschen Vorlage von sich aus formuliert. Das ist ein klares Zeichen dafür, dass die laa- Verdoppelung in Modalkonstruktionen in seinem Dialekt tatsächlich vorkommt. ${ }^{10}$ Diese Gewährsperson ist 72 Jahre alt, auch die Verdoppelungsbeispiele in Modalsätzen in Weber (1948: 247) sind eher älteren Datums. Es könnte die Hypothese aufgestellt werden, dass im Laufe der Zeit mehr syntaktische Restriktionen zur Wirkung kamen. War die Verdoppelung

9 Diese Verteilung zeigt sich auch bei den fünf Übersetzungsaufgaben mit dem Verb ligge. Es ist also ausgeschlossen, dass die Variation an das jeweilige infinite Verb gebunden ist.

10 Zu diesem Argument vgl. Seiler (2005: 329). 
von laa in Modalkonstruktionen vor sechzig Jahren vielleicht noch durchaus üblich, so ist sie heute eher die Ausnahme.

Laut Hypothese 2 würden Nebensatzkonstruktionen die höchste Verdoppelungsrate aufweisen. Auch diese Annahme wird durch die vorliegenden Ergebnisse, wenn auch nur knapp, bestätigt. Dies könnte ein Hinweis auf eine mögliche Funktion der laa-Verdoppelung sein: Das zusätzliche Einsetzen der Kurzform la verhindert ein Aufeinandertreffen von finitem Verb laa und abhängigem Infinitiv. ${ }^{11}$ Ein solches Zusammenkommen ist tatsächlich in keinem der erhobenen Sprachbeispiele zu finden. Wer in den Nebensatzkonstruktionen nicht verdoppelt hat, umging das Aufeinandertreffen von finitem laa und Infinitiv mit der Verwendung der zentripetalen Wortfolge. In den Präsens- und Imperativaufgaben treten jeweils andere Satzglieder zwischen Personalform und Infinitiv.

Diese Korrelation dokumentiert einen Zusammenhang zwischen Wortfolge und Verdoppelung. In den Sprachproben kamen sowohl die zentrifugale wie auch die zentripetale Wortfolge vor. Die zentrifugale (oder aufsteigende) Wortfolge (z. B. la ligge) ist typisch für das Schweizerdeutsche, sie wird in allen Gebieten, ausser in der Nordostschweiz (inklusive des nördlichen Teils des Kantons Zürich), verwendet (cf. Russ 1990: 380 und Glaser 2003: 41). Weber (1948: 247) erwähnt für den Zürcher Dialekt die Möglichkeit der Variation von zentrifugalem und zentripetalem Abfolgemuster in Perfekt- und Modalkonstruktionen, dabei nennt er die zentripetale die weniger gute Variante.

Die Daten des SADS-Projekts zeigen, dass die Grenze bezüglich zentrifugalem und zentripetalem Wortfolgemuster praktisch identisch ist mit der Grenze zwischen Verdoppelungsgebiet und Nicht-Verdoppelungsgebiet. Wie im Abschnitt 1.3 erwähnt verläuft diese Trennlinie durch den Kanton Zürich. Im Westen wird also verdoppelt und das zentrifugale Muster verfolgt. Im östlichen Teil des Kantons wird nicht verdoppelt, und es gilt die zentripetale Wortfolge. Es kann folglich angenommen werden, dass im südwestlichen Teil des Kantons Zürich, woher die vorliegenden Sprachdaten stammen, die zentrifugale Variante die autochthone ist. Das wird dadurch bestätigt, dass diejenigen Gewährspersonen, die diese zentrifugale Wortordnung befolgten, am häufigsten verdoppelt haben.Wer in keiner Aufgabe das Verb laa verdoppelt hat, verwendete ausschliesslich das zentripetale Muster. Dies könnte zweierlei bedeuten: Einerseits entsprechen sowohl die Formulierungen ohne Verbverdoppelung als auch die zentripetaleWortfolge der standarddeutschen Syntax. Möglicherweise liegt hier ein Sprachwandel durch Einfluss der Standardsprache vor. Es kann sein, dass einige Gewährspersonen ihre dialektalen Formulierungen dem Standarddeutschen angeglichen haben und/oder, dass sie sich insbesondere in der Aufgabenstellung des Fragebogens durch die standarddeutsche Vorlage haben beeinflussen lassen.

Da der Kanton Zürich ein Übergangsgebiet bezüglich Verdoppelung und Wortfolge darstellt, wäre es andererseits auch möglich, dass einige Personen ursprünglich gar nie verdoppelt haben und, wie ihre östlichen Nachbarn, schon immer das zentripetale Abfolgemuster verwendet haben. Gegen diese zweite Interpretation spricht allerdings, dass, bis auf die zwei Personen aus Hombrechtikon, alle Gewährspersonen aus dem klar südwestlich liegenden Kantonsteil stammen. Es ist also plausibler, dass die vorliegenden Sprachdaten einen Sprachwandel dokumentieren. Dieser Wandel ist einerseits auf einen standardsprachlichen Einfluss zurückzuführen, andererseits könnte er auch durch Sprachkontakt innerhalb des Schweizerdeutschen entstanden sein. Beim Zusammenstellen der Stichprobe zeigte sich, dass viele Zürichdeutsch-Sprecher und Sprecherinnen keinen einheitlichen Dialekt sprechen, sondern eine Mischung aus den Dialekten ihrer Eltern oder einen Mischdialekt, der aus eigener Migration entstanden ist. Auch Keller (1961: 35) erwähnt die starke Dialektvermischung im

11 Das ist eine Beobachtung, die auch Schmidt (2000: 43) gemacht hat. 
Kanton Zürich. Zusätzlich weist er darauf hin, dass regionale Dialektformen dazu tendieren, sich dem dominierenden Dialekt des Kantons, dem Dialekt der Hauptstadt, anzugleichen. Der Dialekt der Hauptstadt Zürich sei aber wegen dem kosmopolitischen Charakter der Stadt besonders starkem Wandel durch Dialektmischung und Einfluss des Standarddeutschen ausgesetzt. Es ist also anzunehmen, dass im Stadtzürcher Dialekt das Phänomen der laaVerdoppelung aufgrund des Einflusses überregionaler und schriftsprachlicher Strukturen nur noch selten verwendet wird.

Es könnte für das Zürichdeutsche auch angenommen werden, dass immer stärkere syntaktische Restriktionen dazu führten, dass bei Modal- und Perfektkonstruktionen normalerweise die Variante ohne Verdoppelung bevorzugt wird, obwohl den Dialektsprechern in ihrer Grammatik beide Varianten zur Verfügung stehen.

Die Variation in den erhobenen Sprachbeispielen scheint also durch den syntaktischen Kontext gesteuert zu werden. Ebenfalls dokumentieren die Daten den Einfluss durch Sprachkontakt. Andere Hinweise führen zur Variable Alter als weiteren Einflussfaktor.

\subsection{Variation aufgrund des Alters}

Im Abschnitt 3.4 wurde auf eine höhere Verdoppelungsrate bei der älteren Generation hingewiesen. Insbesondere in den Imperativ- und Nebensatzkonstruktionen verwenden die älteren Gewährspersonen deutlich mehr Verbverdoppelungen als die jüngeren. Auch Hypothese 3 kann also bestätigt werden. Die erhobenen Daten können als Anzeichen eines Sprachwandelprozesses interpretiert werden. Die Apparent-time hypothesis (cf. Hofer 1997: 55-57) geht davon aus, dass die synchrone sprachliche Variation zwischen Personen verschiedenen Alters innerhalb einer Sprachgemeinschaft den diachronen Sprachwandel widerspiegelt. Es wird angenommen, dass die beobachtbaren Unterschiede in der Sprache von Sprechern und Sprecherinnen verschiedener Altersgruppen den sprachlichen Veränderungen entsprechen, die sich im Laufe der Zeit herausgebildet haben. Das würde also bedeuten, dass die laa-Verdoppelung früher häufiger verwendet wurde, vor allem in Imperativ- und Nebensatzkonstruktionen. Bei den Präsenssätzen verdoppeln die Altersgruppen ungefähr gleich stark. Diesbezüglich scheint der Sprachwandel weniger weit fortgeschritten. Um die Apparent-time-Hypothese besser $\mathrm{zu}$ stützen, sollten sicher noch mehr Sprachbeispiele von Gewährspersonen unter dreissig Jahren gesammelt werden. Schliesslich aber lässt sich Sprachwandel nur mittels Langzeitstudien der Sprache derselben Gewährspersonen wirklich belegen (cf. Hofer 1997: 57).

Dass eine 35-jährige Informantin in allen sechs Beispielen mit syntaktisch günstigem Kontext verdoppelt hat, weist darauf hin, dass der Sprachwandel nicht nur vom Alter abhängt. Die betreffende Probandin, eine Primarlehrerin, hat immer in Wädenswil gewohnt und auch ihr Mann ist in Wädenswil aufgewachsen. Vermutlich blieben ihr viele ursprüngliche Formen erhalten, da sie den grössten Teil ihres Lebens dem Wädenswiler Dialekt ausgesetzt war. Es scheint von Bedeutung zu sein, wie stark der Kontakt mit anderen Dialekten ist. Dies leitet über zu einer genaueren Betrachtung der regionalen Unterschiede.

\subsection{Regionale Variation}

Die regionale Verteilung der laa-Verdoppelung lag nicht im Fokus dieser Untersuchung. Trotzdem lassen sich aufgrund der erhobenen Daten einige Aussagen dazu machen. Der Dialekt von Wädenswil fällt dadurch auf, dass alle vier Gewährspersonen Verdoppelungskonstruktionen verwendet haben. Es ist möglich, dass hier der Einfluss des Stadtzürcher Dialekts nicht allzu gross ist. Die Stadt Wädenswil liegt $22 \mathrm{~km}$ von Zürich entfernt und bietet selbst ein grosses Angebot an Einkaufsmöglichkeiten und kulturellen Veranstaltungen. Dahingegen mag sich das näher bei Zürich gelegene Dorf Oberrieden mehr nach der Kantonshauptstadt 
ausrichten. Das könnte der Grund für die geringe Verwendung von Verdoppelungskonstruktionen bei den Informanten aus Oberrieden sein.

Eigentlich hätte erwartet werden können, dass im Mettmenstetter Dialekt vergleichsweise viele Verbverdoppelungen zu finden sind. Wie im Abschnitt 2.3 dargelegt, ist im südwestlichen Teil des Knonauer Amtes der Einfluss des angrenzenden Zuger Dialekts festzustellen. Man hätte also vermuten können, dass wie im Kanton Zug auch in Mettmenstetten viel verdoppelt wird. Die Daten zeigen jedoch die tiefste Verdoppelungsrate in dieser Ortschaft. Wie bereits erwähnt hat das Alter der Gewährspersonen die Ergebnisse wohl beeinflusst, darum muss berücksichtigt werden, dass die Altersgruppen ungleich auf die Ortschaften verteilt sind. So setzt sich die Mettmenstetter Stichprobe aus drei jungen Personen zusammen (von 18, 20 und 44 Jahren). Die drei Informanten aus Rifferswil hingegen sind alle über sechzig Jahre alt und ihre Antworten weisen einen hohen Prozentwert der Verdoppelung auf (s. Tab. 2 und Tab. 3).

Weiter muss bei der regionalen Auswertung berücksichtigt werden, dass nicht alle Elternteile der Gewährspersonen den ursprünglichen Dialekt des Ortes sprechen. So wird von Informant 5 aus Mettmenstetten und von der Informantin 10 aus Hombrechtikon jeweils die Mutter als Sprecherin des Berndeutschen angegeben. Die Informantin 12 aus Oberrieden und der Informant 6 aus Mettmenstetten haben je einen Elternteil aus dem Kanton Thurgau. Es ist also schwer zu beurteilen, ob die Antworten vom elterlichen Dialekt beeinflusst sind oder nicht.

Die Anzahl der Gewährspersonen pro Ortschhaft variiert stark. Dies ist ein weiterer Grund dafür, dass die Ergebnisse nicht wirklich einen Vergleich der verschiedenen regionalen Varianten zulassen. Auch sind die Stichproben viel zu klein, um die verschiedenen Ortschaften repräsentativ zu vertreten. Die Variable Region verliert daher bei dieser Untersuchung an Bedeutung. Festgehalten werden kann aber der Befund, dass in allen untersuchten Dialekten Verbverdoppelungen aufgetreten sind. Im südlichen Teil des Kantons Zürich scheinen also die Variante mit Verdoppelung und die Variante ohne Verdoppelung durchwegs nebeneinander vorzukommen. Die Variante ohne Verdoppelung wird dabei mehrheitlich bevorzugt. Die Frage stellt sich nun, in welchem Verhältnis diese beiden Varianten zueinander stehen, welche Funktion die zwei Formen haben könnten (cf. Seiler 2005: 336).

\subsection{Funktion der Verdoppelung}

Um bei zwei syntaktischen Strukturen von Varianten sprechen zu können, müssen diese Strukturen semantisch äquivalent sein, d. h. sie müssen alternative Formen sein, um dasselbe auszusagen (cf. Winford 1996: 180). Zwischen den hier untersuchten syntaktischen Strukturen besteht sicher semantische Äquivalenz. Ob eine Formulierung eine Verdoppelung beinhaltet oder nicht, sie sagt immer dasselbe aus. Es finden sich in der vorliegenden Untersuchung keine Hinweise dafür, dass es sich um eine pragmatisch motivierte Variation handelt. Durch welche Faktoren wird die Variation bedingt? Die erhobenen Sprachbeispiele können nicht viel über die sozialen Konnotationen der Varianten aussagen, da der soziale Status der Gewährspersonen nicht erfasst worden ist. Einzig die Tatsache, dass jüngere Personen weniger verdoppelt haben, könnte ein Hinweis dafür sein, dass Verdoppelungskonstruktionen als altmodisch betrachtet werden. Denkbar wären als externer Steuerungsfaktor auch stilistische Restriktionen (cf. Winford 1996: 181). Möglich wäre, dass unter gewissen äusseren Umständen die Verwendung der mundartlichen Formulierung mit Verdoppelung eingeschränkt wird und die Formulierung ohne Verdoppelung, die sich der Struktur der Standardsprache anlehnt, bevorzugt wird. Diese Mutmassungen lassen sich mit den hier erhobenen Daten allerdings nicht näher untersuchen, lag der Fokus doch auf den syntaktischen Rahmenbedingungen, also den internen Steuerungsfaktoren. 
Ein Hinweis auf die Funktion der Verdoppelung ist denn auch syntaktischer Natur. Wie im Abschnitt 4.1 dargelegt, könnte die verdoppelte Kurzform la die Funktion haben, das Aufeinandertreffen von finitem Verb und abhängigem Infinitiv zu verhindern.

Auffallend ist zudem, dass die Verdoppelungen asymmetrisch auf die je zwei Beispielsätze zu den verschiedenen Satzarten verteilt sind. Die Übersetzungsaufgabe im Präsens, die aus dem Fragebogen des SADS-Projektes übernommen wurde, und ihre Ergänzungsaufgabe im Imperativ dokumentieren deutlich mehr Fälle von Verdoppelung. Diese erste Staffel von Beispielen (Aufgaben 1-5) beinhaltet verschiedene Infinitive, während in den Sätzen der zweiten Staffel (Aufgaben 6-10) immer der Infinitiv ligge verwendet wird. Ich halte es für unwahrscheinlich, dass die Variation vom lexikalischen Verb abhängt, dass sie also semantisch oder phonologisch begründet ist. Ich vermute die Ursache der Variation eher in Richtung der Tatsache, dass die Anzahl der Satzglieder, die zwischen der finiten Form von laa und dem abhängigen Infinitiv stehen, variiert. Während sich in der ersten Aufgabenserie stets nur ein Satzglied zwischen laa und Infinitiv befindet wie in (4a), sind es in der zweiten Serie immer zwei wie in (4b):

(4) a. Er laat de Schriiner choo. (FB 4) / Lönd's sii! (FB 3)

b. Ich lan en immer ligge. (FB 7) / Las en doch ligge! (FB 10)

Eine mögliche Interpretation wäre also, dass das Vorhandensein zweier (oder noch mehr) Satzglieder zwischen der finiten Form von laa und dem Infinitiv die zusätzliche Verwendung der verdoppelten Kurzform la einschränken. Diese Vermutung wäre mit zusätzlichen Erhebungen und verschiedenen Satzbeispielen weiter zu testen.

\subsection{Beurteilung des Erhebungsinstrumentes}

Die Übersetzungsaufgaben schienen den Testpersonen keine grossen Schwierigkeiten bereitet zu haben. Einige Gewährspersonen haben den Testsatz auf Standarddeutsch allerdings zu frei übersetzt. Eine Informantin hat beispielsweise in fast jeder der zehn Aufgaben das Verb lassen durch ein lexikalisch anderes Verb ersetzt. Zum Beispiel:

(5) a. Er lässt den Schreiner kommen: Er brichtet em Schriner. (FB 4)

b. Du kannst ihn ruhig liegen lassen: Chasch en rueig verlege. (FB 8)

Auf die Anfrage hin, ob sie die Sätze auch mit den angegebenen Verben übersetzen könnte, hat sie dies in zwei Fällen getan, in den anderen aber darauf beharrt, eine Formulierung mit dem Verb lassen sei nicht echtes Schweizerdeutsch. Dass einige Personen eine Übersetzung ohne laa + Infinitiv verwendet haben, zeigt aber wohl auch, dass das Erhebungsziel der Befragung für die Gewährspersonen nicht offensichtlich war.

Besonders viele unbrauchbare Antworten gab es bei Aufgabe 5, der ersten Aufgabe zur Nebensatzkonstruktion. Dieser Konjunktionalnebensatz wurde von einem Drittel der Gewährspersonen durch indirekte Rede ohne Konjunktion übersetzt:

(6) Er hat gesagt, dass er den Schreiner kommen lässt.

Er hät gseit, er lösi de Schriiner choo.

Dies ist sicher ein Fehler in der Konzeption der Übersetzungsaufgabe, der durch mehr Pretests hätte vermieden werden können. Insgesamt erweist sich das Erhebungsinstrument als durchaus geeignet zur Erfassung der Verdoppelung des Verbs laa.

\section{$5 \quad$ Zusammenfassung}

Den Sprechern und Sprecherinnen des Zürichdeutschen Dialekts stehen zwei Varianten des Infinitivanschlusses an das Verb laa zur Verfügung: eine Konstruktion mit Verdoppelung von laa und eine ohne. Die interpersonale wie auch die intrapersonale Variation in der Verwen- 
dung dieser beiden Varianten ist dabei sehr gross. Es scheint syntaktische Restriktionen zu geben, die die Verdoppelung von laa einschränken: Ist die Stellung des finiten Verbs im Satz durch ein Hilfsverb oder Modalverb belegt, wird nur äusserst selten verdoppelt. Leicht einschränkend wirken Satzstrukturen mit zwei Satzgliedern zwischen der flektierten Form von laa und dem abhängigen Infinitiv. Günstige syntaktische Rahmenbedingungen für die Verbverdoppelung scheinen hingegen untergeordnete Nebensätze mit der flektierten Form in Endstellung zu bieten. Durch die Kurzform la wird das Aufeinandertreffen vom flektiertem Verb und dem abhängigem Infinitiv verhindert. Ebenfalls günstige Verdoppelungsbedingungen bieten Präsenssätze mit nur einem Satzglied zwischen laa und Infinitiv. Etwas weniger tritt die Verbverdoppelung in Imperativsätzen auf.

In Nebensatz- und Imperativkonstruktionen haben jüngere Personen deutlich weniger die laaVerdoppelung verwendet als ältere. Dies lässt einen Sprachwandelprozess vermuten. Bezüglich der Formulierungen im Präsens war diese Entwicklung weniger gut festzustellen.

Die Verteilung von Verdoppelungskonstruktionen bzw. Konstruktionen ohne Verdoppelung ist weitgehend identisch mit der Verteilung von zentrifugaler bzw. zentripetaler Wortfolge. Dies könnte auch als Anzeichen des Einflusses der neuhochdeutschen Standardsprache gedeutet werden. Sprecher und Sprecherinnen, die nicht verdoppeln und die zentripetale Wortordnung verwenden, könnten diese Strukturen vom Neuhochdeutschen übernommen haben. Um die Funktion der Verdoppelung von laa tiefer zu ergründen und die hier aufgestellten Interpretationen zu testen, sind noch weitere, umfangreichere Erhebungen nötig.

\section{Literatur}

Glaser, Elvira (1997): "Dialektsyntax. Eine Forschungsaufgabe". Bericht über das Jahr 1996. Schweizerdeutsches Wörterbuch. Schweizerisches Idiotikon. Zürich: 11-30. http://www.idiotikon.ch/Texte/Jahresberichte/Id_Jahresbericht_1996.pdf, Stand Jan. 2011.

Glaser, Elvira (2000): "Erhebungsmethoden dialektaler Syntax". In: Stellmacher, Dieter (ed.): Dialektologie zwischen Tradition und Neuansätzen. Beiträge der internationalen Dialektologentagung, Göttingen, 19.-21. Oktober 1998. Stuttgart: 258-276. (= Zeitschrift für Dialektologie und Linguistik Beiheft 109).

Glaser, Elvira (2003): "Schweizerdeutsche Syntax: Phänomene und Entwicklungen". In: Dittli, Beat/Häcki Buhofer, Annelies/Haas, Walter (eds.): Gömmer MiGro? Freiburg, CH: 39-66.

Hofer, Lorenz (1997): Sprachwandel im städtischen Dialektrepertoire. Eine variationslinguistische Untersuchung am Beispiel des Baseldeutschen. Tübingen/Basel: 55-57. (= Basler Studien zur deutschen Sprache und Literatur 72).

Keller, Rudolf E. (1961): German Dialects. Manchester.

Lötscher, Andreas (1993): "Zur Genese der Verbverdopplung bei gaa, choo, laa, aafaa im Schweizerdeutschen". In: Abraham, Werner/Bayer, Josef (eds.): Dialektsyntax. Opladen: 180-200.

Russ, Charles (1990): "High Alemanic". In: Russ, Charles (ed.): The Dialects of Modern German. London: 364-393.

Schmidt, Christa (2000): Die Verbverdoppelung im Zürichdeutschen. Magisterarbeit, Universität Freiburg i. Br.

SDS = Sprachatlas der deutschen Schweiz. Hg. von Rudolf Hotzenköcherle. Bd. III. Bern: 1975.

Seiler, Guido (2005): "Wie verlaufen syntaktische Isoglossen, und welche Konsequenzen sind daraus zu ziehen?" In: Eggers, Eckhard et al. (eds.): Moderne Dialekte - neue Dialektologie. Akten des 1. Kongresses der Internationalen Gesellschaft für Dialektologie des Deutschen (IGDD) am Forschungsinstitut für Deutsche Sprache "Deutscher Sprachatlas" 
der Philipps-Universität Marburg vom 5.-8. März 2003. Stuttgart: 313-341. (= Zeitschrift für Dialektologie und Linguistik Beiheft 130).

Weber, Albert (1948): Zürichdeutsche Grammatik. Ein Wegweiser zur guten Mundart. Zürich.

Winford, Donald (1996): "The Problem of Syntactic Variation." In: Arnold, Jennifer et al. (eds.): Sociolinguistic Variation. Data, Theory, and Analysis. Stanford: 177-192.

\section{Appendix 1: Fragebogen zum zürichdeutschen Dialekt}

Für das Ausfüllen des Fragebogens benötigen Sie etwa 10 Minuten. Alle Angaben werden vertraulich behandelt und dienen ausnahmslos dem Zweck der Dialektforschung.

Herzlichen Dank für Ihren Beitrag!

\section{Angaben zur eigenen Person}

Jahrgang: $19 \quad$ Geschlecht: $\square$ weiblich

$\square$ männlich

Wohnort:

Aufgewachsen in:

Sie sprechen den Dialekt von

Ihre Mutter spricht den Dialekt von

Ihr Vater spricht den Dialekt von

Tel. (für allfällige Rückfragen):

\section{Hinweise zum Ausfüllen}

- Bitte übersetzen Sie bei jeder Aufgabe jeweils den kursiv gedruckten Satz in Ihren Dialekt.

- Schreiben Sie so, wie Sie sprechen würden, ohne Rücksicht auf die Rechtschreibung.

- Damit ich ein möglichst getreues Bild Ihres eigenen Dialekts erhalte, bitte ich Sie, die Fragen alleine zu beantworten und sich nicht beeinflussen zu lassen.

1. Sie sind bei Ihrer Tante zu Besuch. In der Stube hängt eine antike Wanduhr, die früher immer kaputt war. Heute aber läuft sie und schlägt. Erstaunt fragen Sie:

$\rightarrow$ Hast du die Uhr flicken lassen?

2. Marianne und Peter O. sind bei Mariannes Schwester eingeladen. Nach dem Festessen wollen Marianne und Peter helfen, das Geschirr abzuräumen. Die Schwester winkt jedoch ab und sagt:

$\rightarrow$ Ihr dürft alles liegen lassen.

3. Als Marianne und Peter trotzdem mit Aufräumen beginnen, doppelt die Schwester nach:

$\rightarrow$ Lasst es sein! 
4. Brunos Holztreppe ist schon wieder kaputt. Was tut er?

$\rightarrow$ Er lässt den Schreiner kommen.

5. Auch die Nachbarin hat die kaputte Holztreppe entdeckt und fragt, ob Bruno die Treppe nicht reparieren will. Brunos Frau antwortet:

$\rightarrow$ Er hat gesagt, dass er den Schreiner kommen lässt.

6. Marco kehrt nach einem Besuch bei seiner Freundin nach Hause zurück. Nun steht er vor der verschlossenen Haustüre und findet seinen Schlüssel nicht. Wo ist der Schlüssel?

$\rightarrow$ Er hat ihn irgendwo liegen lassen.

7. Marco erzählt seiner Mutter, dass er seinen Schlüssel oft vergisst. Er sagt:

$\rightarrow$ Ich lasse ihn immer liegen.

8. Die Mutter versteckt darauf einen Schlüssel unter der Fussmatte vor der Türe. Sie erklärt Marco, dass es nun kein Problem mehr sei, wenn er den Schlüssel vergisst. Sie sagt:

$\rightarrow$ Du kannst ihn ruhig liegen lassen.

9. Marcos Schwester hat ein ähnliches Schlüsselproblem. Sie trägt den Schlüssel an einem Band um den Hals, ...

$\rightarrow$...weil sie ihn sonst liegen lässt.

10. Maja sieht einen Kaugummi auf der Strasse kleben und will ihn aufheben. Die Mutter sagt:

$\rightarrow$ Lass ihn doch liegen! 


\section{Appendix 2: Karten zur Verbverdoppelung}

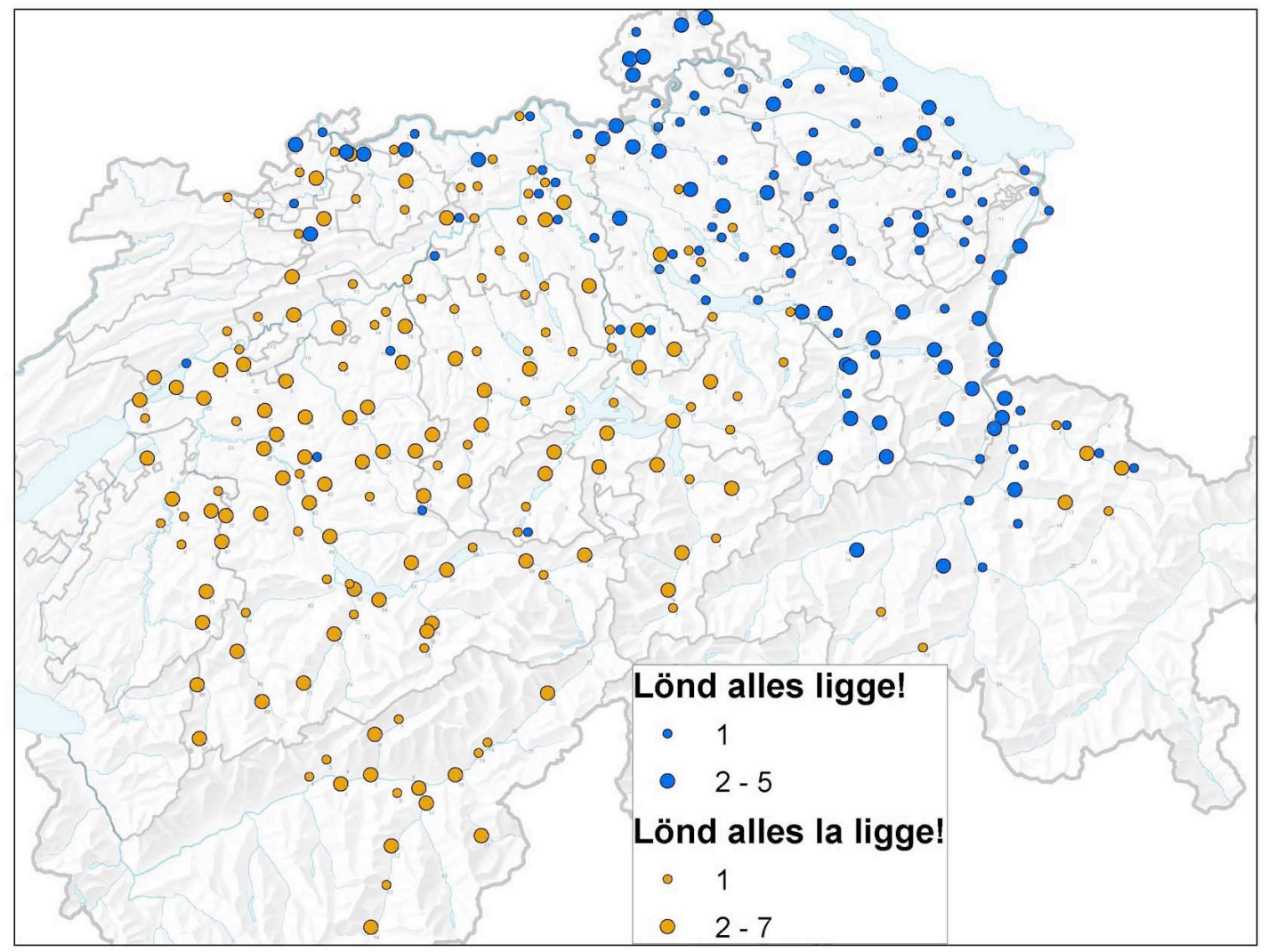

Karte 1: Aufgabe II.5 (SADS) Ihr dürft alles liegen lassen als Imperativ übersetzt (Stand: Dez. 2007)

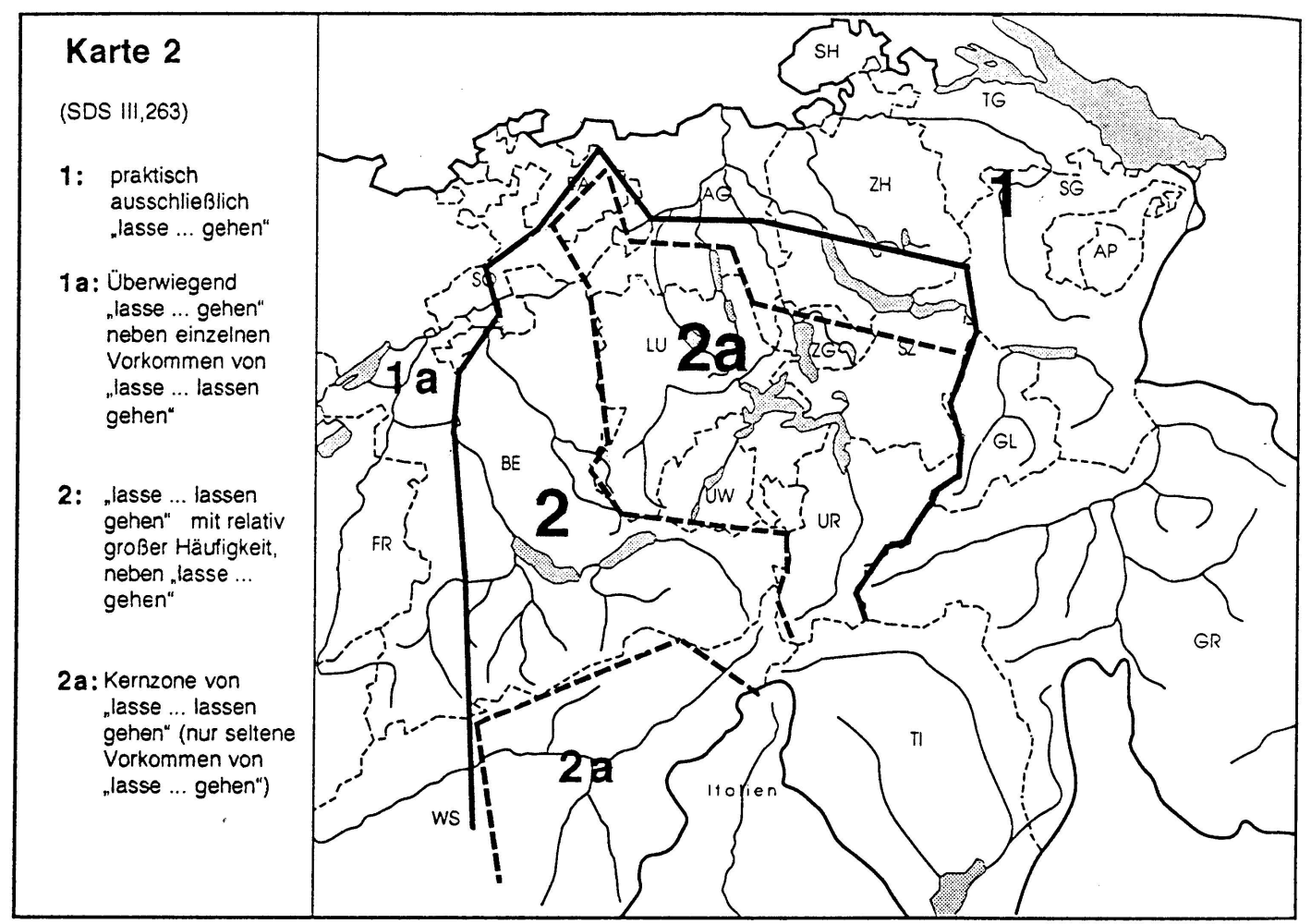

Karte 2: Verteilung der zunehmenden Häufigkeit der Verdoppelung von laa im Imperativ (Lötscher 1993: 196) 